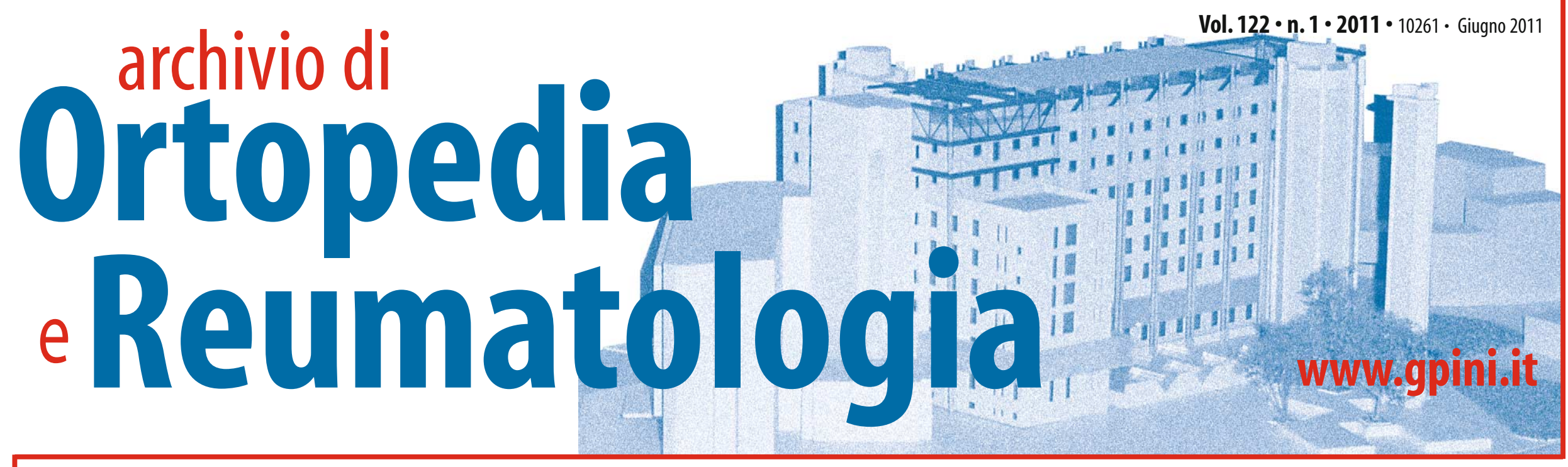

\title{
La traumatologia del cingolo scapolo-omerale
}

a cura di PierLuigi Gambrioli

\section{Editoriale}

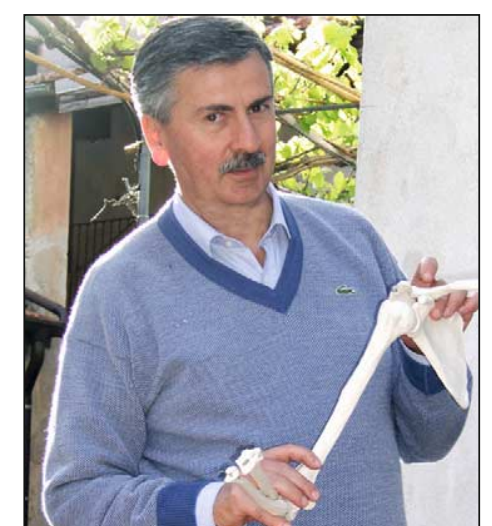

P.L. Gambrioli

a traumatologia del cingolo sca-polo-omerale ha assunto negli ultimi decenni sempre più rilevanza nella attività del traumatologo, sia per l'incidenza che le fratture dell'epifisi prossimale dell'omero hanno in una popolazione sempre più anziana, ma spesso ancora attiva, sia per l'incremento nella popolazione giovane di incidenti sportivi o del traffico ad alta energia.

Ladeguamento delle indicazioni e delle tecniche chirurgiche nel trattamento delle fratture del cingolo scapolo-omerale presso il nostro Istituto è iniziato, quale parte di uno sviluppo moderno della chirurgia della spalla, negli anni Ottanta sotto la guida del Prof. Mario Randelli.

Attraverso la fondazione della Società Italiana di Chirurgia della Spalla e del Gomito, e organizzando regolari corsi di insegnamento, si è cercato di diffondere quanto di valido emergeva a livello italiano e internazionale nella chirurgia e traumatologia della spalla.

Da quell'inizio si sono formate nel nostro Paese un paio di nuove generazioni di chirurghi che si dedicano alla chirurgia e alla traumatologia della spalla e del gomito e oggi possiamo dire che su questo tema è al lavoro un gran numero di specialisti di livello anche internazionale, alcuni dei quali sono stati chiamati a dare il loro contributo in questa circostanza. Questo fascicolo di Archivio di
Ortopedia e Reumatologia è al tempo stesso un bilancio di quanto fatto in passato e il tentativo di delineare ciò che dobbiamo migliorare o rinnovare nella traumatologia della spalla.

Abbiamo quindi voluto riprendere l'analisi delle esperienze accumulate presso il nostro Istituto che in alcune patologie sono state già oggetto di riflessione nelle pagine di questa rivista, sul trattamento delle fratture dell'epifisi prossimale dell'omero allargando l'esposizione ad altri aspetti meno frequenti, ma egualmente problematici, come le fratture di scapola. Uno degli aspetti nuovi nel trattamento della traumatologia della spalla riguarda i progressi delle tecniche mini-invasive e dell'artroscopia nel trattamento delle fratture della piccola e della grande tuberosità omerale isolate, delle fratture articolari della glena nelle quali l'artroscopia ha il vantaggio di evitare esposizioni a cielo aperto con diminuzione dei rischi di rigidità post-operatoria

Nel campo delle fratture a 3-4 frammenti dell'epifisi prossimale dell'omero sono invece stati fatti molti passi avanti nel definire questo gruppo di fratture in modo meno generico di quanto era usuale in base alle classificazioni classiche come quella di Neer. All'interno di questo insieme di fratture sono state individuate quelle a maggiore o minor rischio di necrosi cefalica, quelle stabili o instabili che pongono problemi differenti di riduzione e sintesi vali$\mathrm{da}$, le fratture in valgo o in varo e le fratture su terreno osteoporotico con perdita di "bone stock" osseo, a livello sia della metafisi sia delle tuberosità, differenziandole da quelle su traumi ad alta energia nel giovane. Si è quindi iniziato a proporre tecniche di riduzione sintesi adattate alle differenti tipologie di frattura.

In questa rassegna abbiamo voluto focalizzare l'attenzione soprattutto sulle fratture di maggiore complessità, nelle quali i metodi sinora utilizzati concentrano i loro sforzi per separare gli insuccessi dovuti alle difficoltà intrinseche nel trattamento di alcune fratture da quelli legati a problemi di indicazione o di tecnica chirurgica. Le difficoltà di ricostruzione anatomica di questo tipo di fratture e gli insuccessi da necrosi cefalica restano tuttora un limite che, sperimentando nuove tec niche, si cerca di spostare in avanti, con risultati, ancora oggi, promettenti ma non decisivi.

Lo stesso processo di precisazione classificativa e di adeguamento delle tecniche di trattamento è avvenuto per le fratture di scapola. La relativa rarità delle fratture $\mathrm{d}$ scapola ha impedito a lungo che si potesse concentrare una espe rienza abbastanza ampia per valutare con una metodologia corretta le indicazioni del trattamento conservativo e di quello chirurgico e, nel campo dell'osteosintesi, definire vie d'accesso, materiali di sintesi e, nell'ultimo pe riodo, il ruolo delle tecniche artroscopiche nel trattamento delle fratture con rima a estensione articolare glenoidea.

Dagli anni Ottanta-Novanta sono state individuate tre tipologie $\mathrm{d}$ fratture di scapola: le fratture della superficie articolare glenoidea (isolate o associate a più compless quadri di fratture del collo o del corpo scapolare), le fratture de collo scapolare isolate o associate a "floating shoulder" e le fratture del corpo scapolare, spina scapo- lare, acromion e coracoide senza interessamento del massiccio articolare glenoideo.

Nella realtà clinica le fratture di scapola presentano usualmente una variabile associazione e gravità delle lesioni descritte. Da una review della letteratura sino al 2006 l'80\% delle fratture di glena è stato trattato chirurgicamente con risultati buoni nell' $82 \%$ dei casi e in questo settore l'artroscopia inizia a svolgere un ruolo importante nel trattamento delle fratture del bordo glenoideo, e anche in fratture più complesse. In letteratura l'83\% delle fratture del collo scapolare è stato trattato conservativamente con il $77 \%$ di risultati buoni ed eccellenti. Le "floating shoulder" con lesioni che riguardano due componenti del "superior shoulder suspensory complex" o SSSC di Goss in letteratura sono state trattate conservativamente con buoni risultati nel 94\% dei casi. Il 99\% delle fratture del corpo scapolare può essere trattato conservativamente perché poco o per nulla influente sulla funzionalità della spalla, con l'86\% di risultati buoni o eccellenti.

In questa prospettiva generale, quando la frattura di scapola si presenta nella sua forma più complessa con un'associazione delle differenti lesioni, l'atteggiament attuale è quello di stabilire una gerarchia delle lesioni e trattare quelle che maggiormente influiscono sulla funzionalità. Le fratture di glena devono essere il cen- tro dell'attenzione perché influenzano stabilità e congruenza articolare, le variazioni di orientamento articolare dovute alle fratture del collo scapolare possono alterare in parte la funzionalità sebbene la mobilità muscolare del fulcro scapolare possa compensare entro limiti ampi le variazioni di versione articolare) e le lesioni che riguardano due componenti del "superior shoulder suspensory complex" o SSSC di Goss devono essere spesso trattate chirurgicamente per evitare una instabilità dolorosa locale. Le tecniche di riduzione e sintesi della "floating shoulder" con associate gravi fratture scomposte del collo glenoideo hanno fatto progressi che rendono questa chirurgia meno problematica, con risultati soddisfacenti e quindi applicabili più ampiamente per diminuire la quota di risultati sinora insoddisfacenti.

nfine, anche se gli Editoriali sono molto spesso la parte meno letta di una rassegna, vorrei comunque ricordare che i problem della traumatologia di spalla nascono spesso dall'inadeguatezza del primo approccio diagnostico e di trattamento in pronto soccorso, come accade nelle fratture, lussazioni o fratture-lussazioni posteriori dell'epifisi prossimale dell'omero misconosciute o trattate inadeguatamente sin dalla prima osservazione in pronto soccorso, spesso in soggetti giovani, con continua a pag. 2 


\section{Board Scientifico}

Direttori Scientifici:
M.d'Imporzano,Milano
C.Verdoia,Milano
Vice Direttori Scientifici:
M.Beruto, Milano
S.Brambilla, Milano
Direttore Responsabile:
A. Tropiano, Milano

\section{Comitato Scientifico:}

P. Bartolozzi, Verona - F. Bertoni, Bologna

F. Biggi, Belluno - R. Capanna, Firenze P. Cherubino, Varese - R.D. D'Ambrosia, New Orleans - L. Del Sasso, Como A. Diméglio, Montpellier - W.F.Enneking, Gainesville - F. Fantini, Milano - P.G. Garbagna, Milano - S. Giannini, Bologna A. Lanzetta, Milano - E. Meani, Milano F.Odella, Milano - A. Parafioriti, Milano G. Randelli, Milano - P. Rossi, Torino - C. Verdoia, Milano - V.Zucchi, Milano

\section{Comitato Editoriale:}

S. Caserta, Milano - R. Coluccia, Milano C. Corradini, Milano - C. Cucciniello, Milano - F. Di Domenica, Milano - G. D Luca, Milano - M. Gallazzi, Milano - V. Gerloni, Milano - G. La Maida, Milano - G. Laurà, Milano - F. Maggi, Milano - S. Mapelli, Milano - B. Marelli, Milano - EC. Marinoni, Milano - M. Mazza, Milano - E. Paresce, Milano - L.Sinigaglia,Milano - G. Sironi, Milano - F. Torretta, Milano - S Tosi, Milano A. Ventura, Milano - R. Viganò, Milano

\section{Comitato di Redazione}

W.Albisetti,Milano - M.Berruto,MilanoS. Brambilla, Milano - U. Dacatra, Milano L. de Girolamo, Milano - F. Fischer, Milano P.L. Gambrioli, Milano - L. Pierannunzii, Milano - L.Sinigaglia, Milano - A.Ventura, Milano - S.Zeni,Milano

\section{Segreteria di Redazione:} N.Zerbi, Milano - S.Zeni, Milano

\section{Aziende sostenitrici}

De Puy, Pomezia (RM)

Ceraver, Bologna

Covidien, Segrate (MI)

Lima Lto, Villanova San Daniele del Friuli (UD)

Telea Electronic Engineering, Sandrigo (VI)

\section{Colophon}

\section{Coordinamento editoriale:}

Carlotta d'Imporzano

Elena Bernacchi

Journal Department

Springer-Verlag Italia $\mathrm{Sr}$

Via Decembrio 28, 20137 Milano

Tel.:02 5425 9732/65

Fax:02 54259701

e-mail:

carlotta.dimporzano@springer.com

elena.bernacchi@springer.com

\section{Grafica e impaginazione:}

Graphostudio, Milano

\section{Stampa:}

Grafiche Erredue, Cirimido (CO)

Registrazione del Tribunale di Milano

N. 400 del 24 ottobre 1981

Registro Nazionale della Stampa,

posizione N. 9016

La distribuzione in Italia avviene

ai sensi della legge 675/96

Springer fa parte di

Springer Science + Business Media

springer.com

\section{springer.com \\ OSpringer-Verlag Italia, 2011 \\ Via Decembrio 28, \\ 20137 - Milano, Italia \\ Stampato in Italia \\ Versione elettronica \\ e realizzazione Web: \\ Anna Gallicchio \\ smm Srl - Scientific Multimedia \\ http://www.smm-srl.it \\ Corso Vercelli 9 - 20144 Milano \\ e-mail:a.gallicchio@smm-srl.it}

\section{Abbonamenti 2011}

ISSN 0390-7368 (versione cartacea)

ISSN 1592-7113 (versione elettronica) $€ 83,00$ (4 fascicoli),

inclusa la versione on-line

Gli ordini dovranno essere indirizzati a Springer-Verlag Italia Srl

Journal Department

Via Decembrio 28

20137 Milano

e-mail: elena.bernacchi@springer.com

\section{Springer}

la rivista on-line

Vieni a visitarci all'indirizzo http://www.gpini.it User ID: arch2001

Password: pini

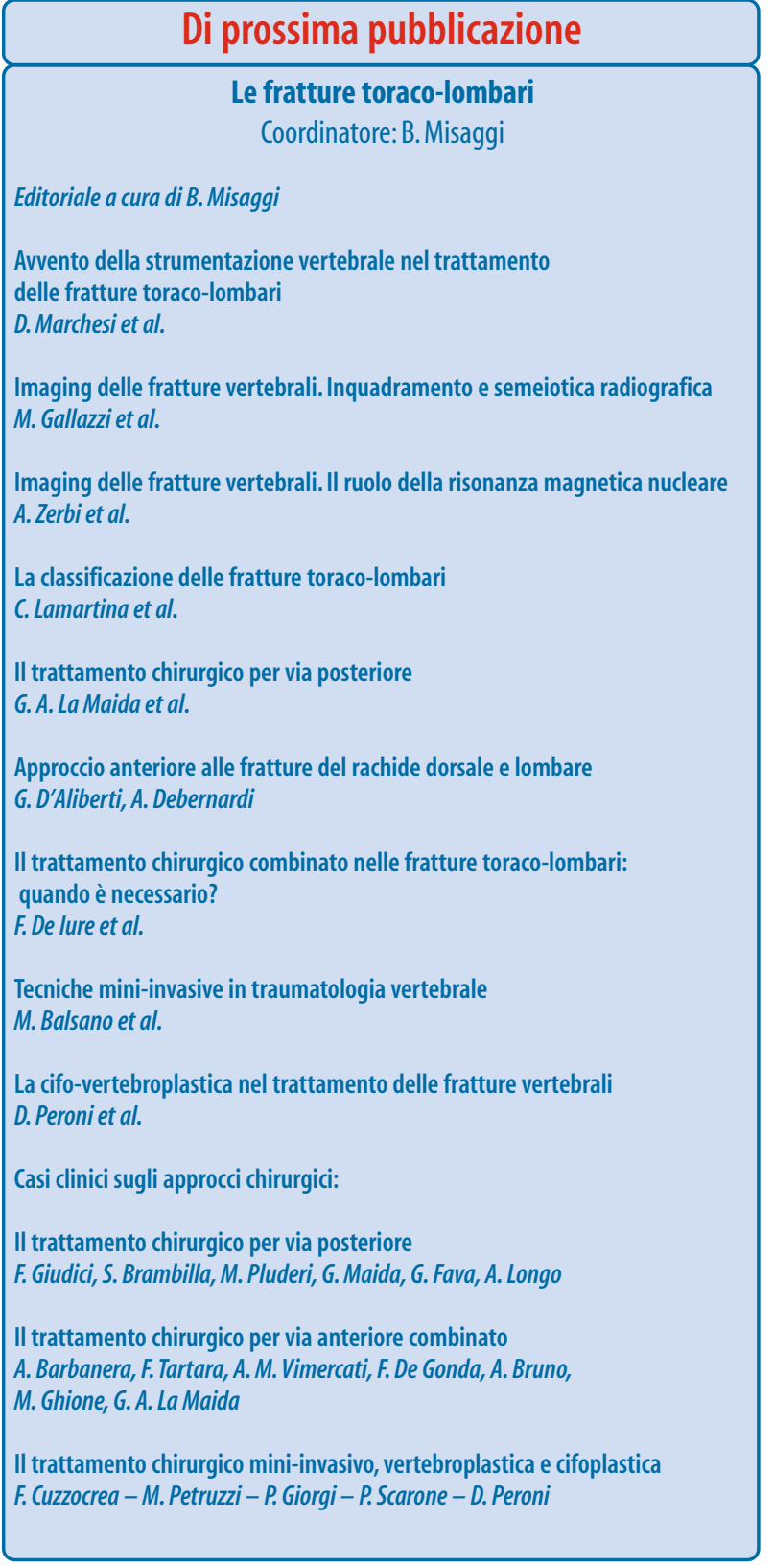

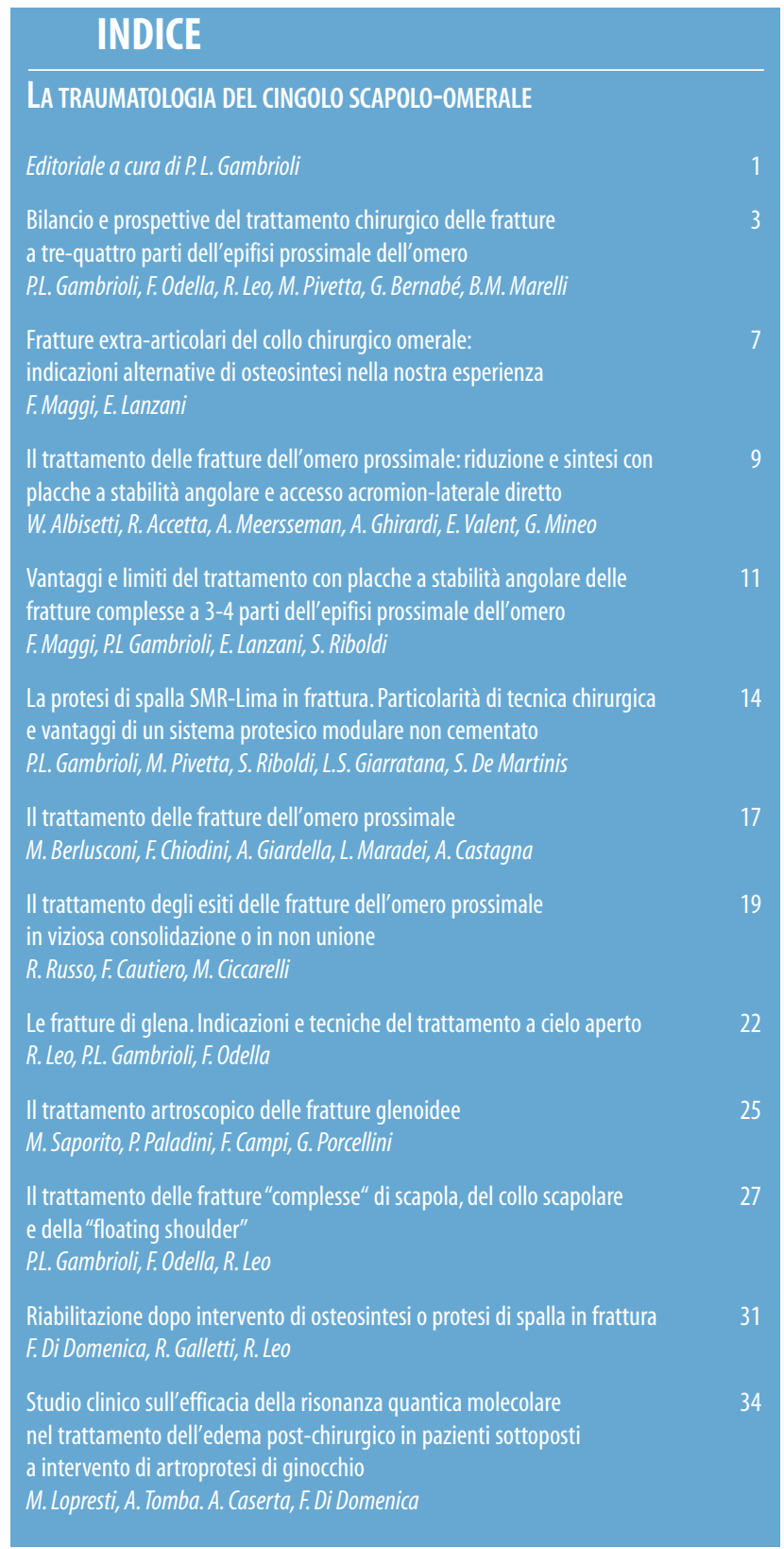

\section{$\nabla$ Editoriale} conseguenze gravi. Partendo dalla diagnostica strumentale, la diagnostica offre oggi in qualsiasi situazione locale almeno due livelli: quello più immediato di un esame radiografico tradizionale e la possibilità, immediata o appena differita, di una TAC che nei casi complessi può fornire anche una ricostruzione tridimensionale.

Con questi esami si possono evitare le lussazioni posteriori o anteriori misconosciute inveterate e le fratture scomposte di trochite e piccola tuberosità inveterate con retrazione del muscolo sottoscapolare che, oltre al contenzioso medico-legale che alimentano, sono più semplici da trattare se diagnosticate immediatamente.

Ritengo inoltre indispensabile una TAC in tutti i casi di fratture a 34 parti dell'epifisi prossimale del-

l'omero che radiologicamente

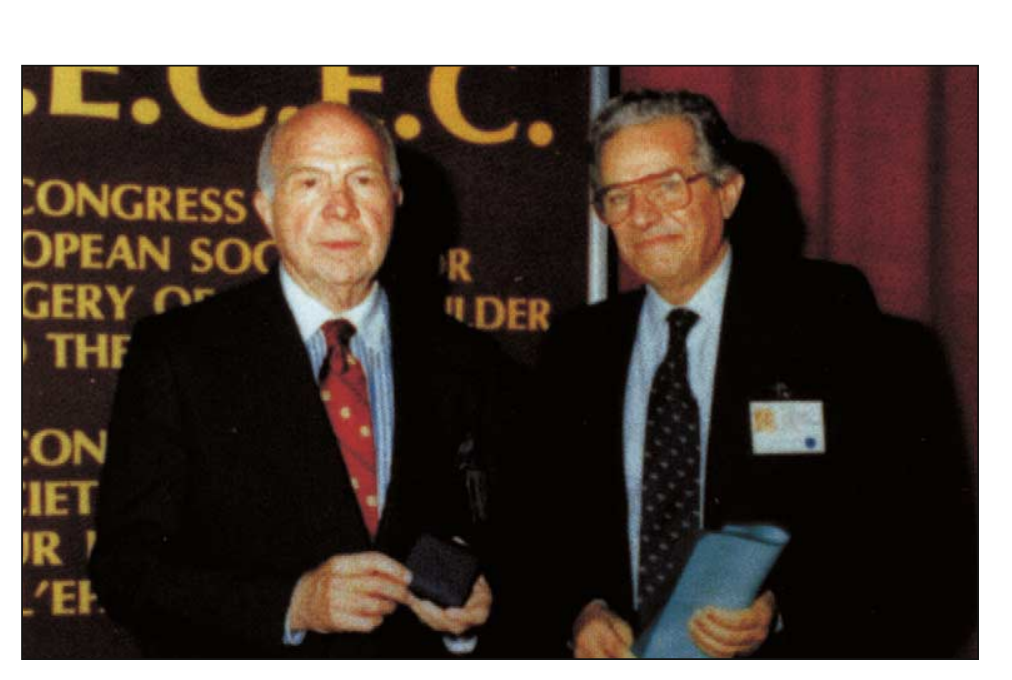

Due tra i padri fondatori della moderna chirurgia della spalla: C.S. Neer (a sinistra) e M. Randelli nel 1990

sembrano abbastanza composte da essere trattate conservativamente, per individuare eventuali fratture della testa omerale e scomposizione delle tuberosità che sul piano assiale possono limitare meccanicamente la rotazione della testa omerale.

Un'altra patologia molto più grave che colpisce in eguale misura soggetti giovani ancora in età lavorativa e soggetti anziani è quella relativa alle lussazioni glenoomerali anteriori traumatiche, molto spesso con frattura del trochite, nelle quali non si riconosce radiologicamente la presenza di una frattura composta del collo anatomico e, nella manovra di ri duzione della lussazione, si ha un distacco-lussazione anteriore della testa omerale. In questi pazienti il rischio di necrosi cefalica dopo riduzione e sintesi è del 60 100\% (secondo che siano conservate o meno connessioni capsula- ri della testa) e il trattamento con protesi di spalla in un soggetto giovane può essere soddisfacente sul breve periodo, ma presenta complicazioni e insuccessi sul medio e lungo periodo.

Due regole sono quindi fondamentali. Innanzitutto non si deve mai procedere a riduzione "d'urgenza" di una lussazione anteriore traumatica di spalla senza avere una radiografia. La radiografia pre-riduzione deve essere valutaa attentamente e, in caso di dubbio sulla stabilità delle connessioni ossee a livello del collo anatomico dell'omero, si deve esaminare la frattura con una TAC e la riduzione deve essere eseguita in sala operatoria in narcosi e sotto controllo in brillanza.

Come ritengo emerga da questa introduzione, la traumatologia de cingolo scapolo-omerale pone ancora affascinanti sfide per i traumatologi e i chirurghi della spalla. Accanto alle tecniche e ai materiali di sintesi, si attende un cambiamento di prognosi, soprattutto per quanto riguarda pseudoartrosi o necrosi vascolare, dallo sviluppo di sostituti dell'osso, fattori di crescita ossea e stimolatori di una neo-angiogenesi nel tessuto devascolarizzato, che ancora di utilizzo sperimentale, possono costituire l'elemento di svolta nella prognosi di queste fratture.

P.L. Gambrioli S.S. Dipartimentale di Chirurgia e Patologia della Spalla e del Gomito, II Divisione di Chirurgia Ortopedica e Traumatologia, Istituto Ortopedico G. Pini, Milano 